\title{
« Tout est sauvage et splendide, comme en Eden »
}

L'œuvre d'Ivan Bounine et l'exotisme

\section{Olga Berdnikova}

Traducteur : Andrea Cantinotti

\section{Q OpenEdition}

\section{Journals}

Édition électronique

URL : http://journals.openedition.org/edl/425

DOI : $10.4000 /$ edl. 425

ISSN : 2296-5084

\section{Éditeur}

Université de Lausanne

\section{Édition imprimée}

Date de publication : 15 septembre 2009

Pagination : 187-202

ISBN : 978-2-940331-20-8

ISSN : 0014-2026

\section{Référence électronique}

Olga Berdnikova, « «Tout est sauvage et splendide, comme en Eden » ", Études de lettres [En ligne], 2-3 | 2009, mis en ligne le 15 septembre 2012, consulté le 20 décembre 2020. URL : http:// journals.openedition.org/edl/425; DOI : https://doi.org/10.4000/edl.425 


\section{"TOUT EST SAUVAGE ET SPLENDIDE, COMME EN EDEN": L'CEUVRE D'IVAN BOUNINE ET L'EXOTISME}

Prosateur et poète, l'un des plus grands stylistes de la littérature russe, Ivan Bounine fait souvent appel dans son œuvre aux images exotiques. Ces images sont ici examinées, depuis celles que lui inspire le monde du Proche-Orient, ce monde dont parle la Bible, jusqu'à celles que l'écrivain glane lors de ses nombreux voyages, dans ses rencontres avec les mœurs, la pensée, les religions, des pays lointains, tel le bouddhisme. L'article s'attache à montrer comment Bounine arrive à rendre le lointain proche et l'exotique, familièrement «originel» et primordial.

La question de l'exotisme dans la littérature russe a peut-être été posée en premier par Iouli Aïkhenvald, dans son introduction à la publication de Silhouettes d'écrivains russes (1911). Le critique y propose un «schéma pour l'étude de la littérature russe» tout à fait original, ayant pour fondement l'hypothèse de la "lutte de deux principes» dans la littérature et dans «le destin social» de la Russie. Il les qualifie:

[...] de noms différents, mais dans leur profondeur apparentés: c'est - la langueur de la patrie et la langueur de l'étranger (terme de Kant), les forces centripète et centrifuge, l'état statique et l'état dynamique, la sédentarité et le nomadisme, le patriotisme et le cosmopolitisme, et, dans le débat social en Russie, l'occidentalisme et le slavophilisme ${ }^{1}$.

Observant les notions de "patrie» et d' "étranger" dans la vie et l'œuvre des écrivains russes du début du $\mathrm{XX}^{\mathrm{e}}$ siècle, Aïkhenvald découvre dans l'œuvre poétique de trois poètes de ses contemporains, Ivan Bounine,

I. J. Ajhenval'd, Siluèty russkih pisatelej, p. 30 (souligné par l'auteur). 
Konstantin Balmont et Nikolaï Goumilev, l'opposition de «l'exotisme et de la patrie». Ces auteurs représentent trois différentes orientations esthétiques et stylistiques de la poésie russe de l'Age d'argent. Il est remarquable que, selon le critique, leur seul point commun se révèle l'exotisme (èkzotika) - qui apparaît comme le résultat de leurs voyages au Moyen-Orient et à Ceylan, au Japon et en Afrique.

Notons que "l'étranger» des poètes du début du XXe siècle était, des points de vue géographique et ethnographique, beaucoup plus exotique que "l'Italie» de Gogol, la "Nice» de Tiouttchev et même que le "Caucase» de Pouchkine et Lermontov, qui étanchait, comme l'observe avec finesse Aïkhenvald, la soif exotique des poètes russes au XIX ${ }^{\mathrm{e}}$ siècle. Au début du XXe siècle, la culture russe a découvert «l'Orient» comme un concept complexe et volumineux qui intégrait diverses «coordonnées" géographiques, historiques, religieuses, esthétiques; par ailleurs, «l'Orient» se révélait un tout et, sous ce rapport, était à l'opposé de «l'Occident». Dans la tradition de la philosophie russe, «l'Orient» a été plus d'une fois le prétexte à une interprétation de la mission providentielle, historique et nationale de la Russie en tant que pays eurasien; à la charnière des $\mathrm{XIX}^{\mathrm{e}}$ et $\mathrm{XX}^{\mathrm{e}}$ siècles, il devient l'une des sources qui nourrissent l'«idée russe», ou une certaine idée de la russité.

En même temps, l'esthétisme devient la tendance dominante dans la culture et la littérature. Un intérêt purement artistique se développe envers l'Orient, lieu de l'exotique, capable de satisfaire la quête esthétique de cette "beauté nouvelle», pour laquelle l'artiste est prêt à «abattre toutes les barrières" et à "transgresser toutes les limites" (Dmitri Merejkovski). C'est ce qui rend possible l'apparition dans la poésie de Goumilev de cette "élégante girafe», qui erre «bien loin sur le lac Tchad»- une image artistique précieuse en elle-même. "L'exotique» signifie bien, selon la définition de Dahl, un phénomène naturel originaire d'ailleurs, "de pays chauds" de préférence ${ }^{2}$. Cependant, en règle générale, conformément au principe de la créativité mythologisante propre à l'esthétique de l'Age d'argent, les images exotiques "pures» sont en même temps chargées de significations religieuses, philosophiques, mythiques, historiques et autres. Apparaissent des phénomènes artistiques particuliers, marquant l'époque et ses différents créateurs et qui se

2. V. Dal', Tolkovyj slovar' živogo velikorusskogo jazyka, p. 663. 
caractérisent par leur appartenance initiale à une autre sphère ethnique, géographique et culturelle. Ainsi naissent «l'Afrique» de Goumilev et de Vélimir Khlebnikov, «l'Egypte» de Balmont, «l'Inde» du peintre et mystique Nikolaï Roerich, «le Turkestan» du peintre Pavel Kouznetsov. La liste n'est évidemment pas exhaustive de ces créateurs qui, selon l'expression de Khlebnikov, "se tourmentent" d'Orient dans la quête des profondeurs de l'antique sagesse, des sources des cultures et civilisations, de la proximité religieuse et ethnique avec la Russie, "du primitivisme» comme d'un nouveau style dans l'art.

Et dans leur lignée, semble-t-il, une place de droit revient à Bounine, car dans sa personnalité et dans sa conscience créatrice se réunit tout ce qui prédispose à l'exotisme. Il est doté d'un goût artistique "unique», «incroyablement fin ${ }^{3}$, qui lui permet «de scruter la beauté et de laisser après lui une ciselure de son âme» " ${ }^{4}$. Les dons poétiques de Bounine l'inclinent à la représentation de la nature, si bien qu'il donne même une «justification" poétique à la critique du début du $\mathrm{XX}^{\mathrm{e}}$ siècle:

Non, ce n'est pas le paysage qui m'attire,

Le regard assoiffé ne remarque pas les couleurs,

Mais ce qui dans ces couleurs luit:

L'amour et la joie de l'existences.

Et, enfin, selon l'affirmation de Maxime Gorki, Bounine est entraîné par «une gravitation organique et héréditaire vers l'Orient " ${ }^{6}$, qu'il suit dans ses voyages au Proche-Orient et à Ceylan et dans ses créations poétiques ainsi qu'en prose. Par conséquent, Bounine se présente comme le prétendant idéal au rôle de poète exotique principal dans la poésie russe.

Le thème "Bounine et l'Orient» est déjà formulé par Olga Slivitskaïa dans les années 1970 et étudié par de nombreux chercheurs; cependant, l'«Orient» ou "Ceylan» chez Bounine ne sont pas vraiment vus comme de l'«exotisme». Il est vrai que «l'Orient» bouninien n'est pas homogène,

3. K. I. Čukovskij, «Smert', krasota i ljubov' v tvorčestve I. Bunina», p. 48.

4. I. Bunin, Sobranie sočinenij v 6 tt., t. III, p. 500 : «[...] obozret' krasotu i ostavit' po sebe čekan duši svoej». Désormais, les chiffres romains et arabes en notes infrapaginales renvoient au volume et à la page de cette édition.

5. I, p. 101: «Net, ne pejzaž vlečet menja, / Ne kraski žadnyj vzor podmetit, / A to, čto v ètih kraskah svetit: / Ljubov' i radost' bytija».

6. M. Gor'kij, Sobr. soč. v 30 tt., p. 146. 
tout comme l'orientalisme russe du début du $\mathrm{XX}^{\mathrm{e}}$ siècle, dominé par la conception des «deux Orients» formulée ainsi par Vladimir Soloviev:

O Russie! Dans une vision élevée

Tu es saisie d'une pensée fière;

De quel Orient veux-tu être,

L'Orient de Xerxès ou celui du Christ? ${ }^{7}$

Dans les années 1900-1910, cette célèbre question ne se pose pas à Bounine sous forme d'alternative: "l'Orient de Xerxès" l'intéresse, tout comme "l'Orient du Christ». La langueur de l'étranger, que Iouli Aïkhenvald avait notée chez lui, le mène avant tout vers «l'Orient du Christ", qui trouve son incarnation dans le cycle prosaïque L'Ombre de l'oiseau (Ten' pticy, 1907-1911). Les récits de ce cycle s'apparentent à des carnets de voyage, ce qui témoigne de la valeur intrinsèque que prend pour l'écrivain le voyage dans le Proche-Orient. Toutefois, Bounine ne considère les pays et Etats qu'il traverse qu'en relation à leur appartenance au monde de la Bible:

Quel paradis, le Liban... Le monde d'Abel! Le monde de la foi pure des enfants! ${ }^{8}$

C'est précisément dans la "géographie» que Bounine voit une valeur esthétique: les pérégrinations sur les lieux bibliques apparaissent simultanément comme un déplacement dans l'espace et dans le temps. "Oui, tout est ici plus ancien, plus oriental ${ }^{9}$, ce qui veut dire: plus c'est oriental, plus c'est ancien; plus c'est ancien et plus c'est poétique. La réalité géographique est pour ainsi dire "reconnue», "remémorée» par l'écrivain voyageur, parce qu'elle s'est déjà révélée objet de représentation artistique dans la Bible, dont les citations, motifs et images sont si présents dans les récits du cycle. "Y a-t-il au monde une autre terre où seraient rassemblés tant de souvenirs chers au cœur humain ?» ${ }^{10}$. De

7. «O Rus'! V predviden'i vysokom / Ty gordoj mysl'ju zanjata: / Kakim Ty hočeš” byt' Vostokom, / Vostokom Kserksa il' Hrista?» ("Ex Oriente Lux», 1890).

8. I, p. 233.

9. III, p. 519: «Da, vse tut staree, vostočnee.»

Io. III, p. 561 : «Est' li v mire drugaja zemlja, gde by sočetalos' stol'ko dorogih dlja čelovečeskogo serdca vospominanij?» 
là vient que l'authenticité des lieux entourant l'auteur et narrateur soit en même temps la preuve principale de leur importance artistique et esthétique, et la précision dans la description de l'itinéraire du voyage, la condition indispensable à la reconstitution poétique de la réalité. La Terre sainte était en elle-même la source du poétique, elle qui garde des "traces» tangibles - géographiques, historiques et proprement sacrées de la vie terrestre du Christ, des prophètes bibliques et des ancêtres vétérotestamentaires, ainsi que des peuples et civilisations les plus anciens. Ce n'est pas par hasard que Bounine souligne si souvent l'authentique antiquité de tout ce qu'il voit autour de lui: du désert, où le Christ fut tenté, de «la terre rouge», de laquelle fut «créé Adam», des bois sacrés de l'Eden "aux Cèdres du Liban au-delà des nues». Son voyage devient la «matérialisation de la Bible» ${ }^{11}$.

Aussi, la simplicité de la vie et des us des peuples de l'Orient biblique, primitive pour un Européen civilisé, est-elle perçue par l'auteur-narrateur non comme un exotisme, mais comme un "état originel». Cette notion acquiert, dans le contexte du cycle et de toute l'œuvre de Bounine, une connotation positive, ce dont témoigne la correction manuscrite par Bounine de la nouvelle "La Lance du Seigneur» («Kop’e Gospodnee»), où le mot «originel» (pervobytnaja) est corrigé en "vétérotestamentaire» (vethozavetnaja) ${ }^{12}$. L'Ancien Testament est garant de l'antiquité, de l'authenticité, de l'essence des fondements de la vie humaine. Ainsi, en voyant des pêcheurs transporter des voyageurs sur le lac, vers Capharnaüm, l'auteur et narrateur cite les versets de l'Evangile sur les premiers apôtres Pierre et André et s'exclame: «Ne pouvait-Il pas aussi appeler ceux-ci?» ${ }^{13}$.

Cela dit, le regard de Bounine européen se révèle différent dans sa perception des manifestations païennes de la "pauvreté originelle» - en ce sens pleinement exotique - lors de la description "de cahutes de glaise à demi animales" et de l'habitant de ces cabanes, "du fellah", qui est comme sorti de la profondeur des siècles:

[...] c'est un taureau accompli, par sa constitution inhumaine, avec son cou de bronze à la force prodigieuse. Et il s'assied comme il lui

II. V. N. Muromceva-Bunina, Žizn' Bunina. Besedy s pamjat'ju, p. 309.

I2. Manuscrit avec les corrections d'auteur.

I3. III, p. 585. 
plaît, à lui le descendant direct des anciens Egyptiens: droit, d'un calme inhumain, les épaules relevées, en posant précisément ses paumes sur ses genoux... ${ }^{14}$

On observe, même dans ce cas, une poétisation et une sacralisation de "l'état originel», retrouvé dans le contexte d'une autre tradition culturelle et cultuelle: cette image rappelle directement la statuaire des sphinx égyptiens.

Ainsi, la réalité de «l'ailleurs", la nature, la vie quotidienne, sont pour ainsi dire «remémorées». La conscience de l'auteur-narrateur les expérimente comme sacrées, les valorise en tant que manifestations du culte et de la culture et, précisément pour cette raison, les accueille en tant qu’appartenant à sa "patrie» spirituelle. Elles ne sont pas perçues en tant qu'un ailleurs exotique. Le voyage devient en grande partie un pèlerinage en Terre sainte, plutôt qu'un déplacement en des contrées exotiques.

Quant à l'« Orient de Xerxès ", celui-ci se présente chez Bounine avant tout sous la forme de l'"Orient du Bouddha».

L'intérêt de l'écrivain pour le bouddhisme est bien connu, et les motifs et images bouddhistes suffisamment étudiés dans son œuvre de jeunesse ${ }^{15}$. Thomas Marullo tente même de présenter l'écrivain comme un quasi-bouddhiste, ce qui serait, si c'était vrai, le plus grand exotisme dans l'histoire de la littérature russe ${ }^{16}$.

Le bouddhisme fascine réellement Bounine en tant que doctrine religieuse et philosophique, dont la base est formée par des idées proches de sa vision du monde: la soumission de l'être humain à la puissance des désirs et passions terrestres ainsi que la possibilité de surmonter cette servitude; l'homme comme partie du monde naturel, cosmique; une représentation cyclique du temps. Cependant, cette relation au bouddhisme revêt chez Bounine un caractère plus artistique que philosophicoreligieux. Cela apparaît pleinement dans le récit "La Nuit» ("Noč"), où, à l'aide de notions bouddhistes, il étaye sa conception de la Mémoire créative, ainsi que dans le livre La Libération de Tolstoï (Osvoboždenie

I4. III, p. 526.

I5. O. Slivickaja, "Povyšennoe čuvstvo žizni»; E. Smol'janinova, «Vostočnye motivy v rannej proze I. A. Bunina»; Tjan’ Hunmin', "Sopostavlenie rasskazov "Sosny" i "Brat'ja" s točki zrenija buddizma», etc.

I6. T. G. Marullo, «If You see Buddha». 
Tolstogo, 1937) qui situe la personnalité artistique de Léon Tolstoï au croisement des traditions bouddhistes et chrétiennes.

On trouve les lieux exotiques de l'Orient bouddhique (et, plus rarement, musulman) - Ceylan (et l'Inde), la Perse, la Chine, le Sahara, la Turquie, Alger - dans bien des écrits appartenant à des époques différentes, mais leur symbolique «culturologique» est finalement peu liée à des manifestations exotiques à proprement parler. Par ailleurs, le monde bouddhique en tant qu'Orient exotique apparaît dans les nouvelles et poèmes bouniniens des années 1910-1920, composés à la suite du séjour à Ceylan en $1911^{17}$.

Ceylan (et l'Inde) est perçu avant tout par lui comme la «terre des ancêtres", les lieux paradisiaques d'Adam et d'Eve, où, avec une puissance tropicale particulière, se refléta la perception si propre à Bounine de la plénitude constante et inchangée de l'existence, de la force vitale comme tentation. Dans la nouvelle "Frères» (1914) deviennent particulièrement significatives les images de midi et de la nuit, qui symbolisent le pouvoir «du dieu de la vie-mort Māra, dieu de la "soif de l'existence" " ${ }^{18}$. Dans "la canicule de midi» meurt le vieux pousse-pousse, et «avec la nuit à la chaleur particulière» revient dans un perpétuel cycle naturel le jeune pousse-pousse.

L'expérience exotique de Bounine voyageur dans l'Orient du Bouddha révèle pour lui la véracité, au sein de la nature, des symboles du christianisme et du bouddhisme, deux religions du monde; et Bounine matérialise cette symbolique dans son ouvre. Ainsi, la canicule de midi dans son monde artistique apparaît comme un motif de la tentation, et par voie de conséquence, le hâle ou le teint bronzé de la peau devient dans la description bouninienne l'un des attributs de la féminité ("En chemin», "Coup de soleil», «La Vengeance», "Au printemps, en Judée» et bien d'autres). Chez lui, la femme s'imprègne, comme de l'héritage du péché originel, de cette "chaleur de midi», dans laquelle, selon la tradition, «le démon de midi séduisit Eve» ("Evu poludennyj bes iskusil»: "Chaleur de midi»/ «Poludennyj znoj», 1947). C’est la raison pour laquelle le «thème» asiatique et, en particulier, indien se matérialise souvent chez

I7. «Frères» («Brat'ja»), «Les Rêves de Tchang» («Sny Čanga»), «La Nuit du renoncement» («Noč” otrečenija»), "Un Compatriote» ("Sootečestvennik»), «Hotami» («Gotami») et quelques autres.

I8. IV, p. 9: «boga Mara, boga žaždy susčestvovanija». 
Bounine dans l'image féminine: l'héroïne est souvent semblable à une Indienne, ce qui renvoie aux motifs du mystère, de l'antiquité, de l'incompréhensibilité du charme séduisant de la femme (Cent roupies, Camargue, La Vie d'Arseniev, Un Lundi pur, etc.).

Par ailleurs, le motif de la tentation, lié à l'image de midi/nuit, a dans la prose de Bounine une origine tropicale, car sous les tropiques uniquement minuit ne le cède en rien à midi en saturation d'énergie vitale et en éclat des coloris:

La nuit éteignit rapidement les couleurs de conte tendres, roses et vertes du crépuscule expirant..., et les forêts s'emplissaient de ténèbres noires et chaudes, s'embrasant de myriades de mouches luisantes et mystérieusement, dans la torpeur, résonnant de couleurs, dans lesquelles vivent de petites grenouilles d'arbres ${ }^{19}$.

Dans un récit plus tardif, peut-être le plus bouddhiste de Bounine, "La Nuit du renoncement» (1921), c'est justement la nuit tropicale qui devient pour l'auteur-narrateur la source de profondes méditations et développements philosophiques sur la force tentatrice des attachements et passions terrestres de l'homme, sur la séduction par la beauté et la «suavité» de l'existence terrestre.

En même temps, une sorte de discours colonial se laisse déceler chez l'écrivain («Frères», "Un Compatriote», «Le Fils»), lorsqu' il décrit la vie des Européens dans un Orient exotique; presque inévitablement, de telles descriptions véhiculent la problématique Occident-Orient de la civilisation et de la nature et, par conséquent, la représentation de l'exotique des "pays chauds». L'exotisme est porté alors par des lieux inhabituels, des phénomènes précisément nommés du monde animal et végétal, des particularités de cette image coloniale de la vie d'Européens et d'Indigènes. Pourtant, même ici, la position de l'auteur-narrateur se présente avec ambiguïté: "l'état originel" se révèle préférable à la civilisation, préférence qui se reflète dans le style de la narration. Ainsi, dans la nouvelle «Frères», les événements sont présentés comme s'ils étaient perçus du point de vue des habitants des tropiques (surtout dans la première partie), ce qui neutralise quelque peu la couleur

19. IV, p. 9: «Noc’ bystro gasila skazočno-nežnye, rozovye i zelenye kraski minuvših sumerek [...], i černoj žarkoj t'moj napolnjalis' lesa, zagorajas' miriadami svetjaščihsja muh i tainstvenno, znojno zvenja cvetami, v kotoryh živut melkie drevesnye ljaguški ». 
exotique. Bounine souligne le côté organique, naturel de la vie et de la mort du vieux et du jeune pousse-pousse. Tentés par la «soif d'amour et de nouveaux enfantements", ils sont en conséquence voués à la mort comme à un retour dans le processus du cycle de la nature, donc inévitablement condamnés à une renaissance dans une autre condition, dans la Roue du Samsâra. En ce sens, la figure de l'Anglais à demi mort, à demi endormi, apparaît bien plus exotique que celles des Indiens. Avec ses yeux "étranges», comme aveugles, ayant «survécu à ses désirs», il vit par inertie, incapable d'être séduit ni par la beauté exotique de l'Orient ni par la "soif d'amour", saisi par la peur de se dissoudre dans "cette terrible Pan-Unité» et d'y perdre sa «Personnalité».

De même, dans la nouvelle "Frères", l'aspect colonial et l'exotisme au sens propre sont surmontés par l'idée de fraternité: Bounine montre non seulement des «frères qui se battent l'un l'autre», comme le déclare l'épigraphe, mais aussi des "frères» unis dans la vanité de leurs prétentions humaines, dans leur indigence spirituelle. Sous cet angle, ni le naturel ni la civilité ne deviennent pour l'écrivain des critères de l'individu. Le côté naturel du Cinghalais, le côté civilisé du Britannique ne les empêchent pas d'être "frères" en ce que l'un et l'autre sont sourds à la voix du Très-Haut, avec la seule différence que le premier ne s'en rend pas compte, alors que l'Anglais est lui capable de réflexion. Pour l'écrivain, dans ce cas précis, les différences confessionnelles n'ont pas d'importance, étant donné qu'il considère la foi comme une nécessité ontologique de l'homme au même titre que le pain quotidien - si bien que les doctrines bouddhiques ne lui apparaissent pas comme véritablement exotiques.

Dans la nouvelle "Un Compatriote», l'écrivain montre un Russe, vivant sous les tropiques, qui sent se réveiller en lui la mémoire génétique de l'ancien Orient qu'il voit du coup comme sa "patrie». C'est précisément les tropiques qui ont révélé au paysan Zotov la possibilité d'«éprouver des sensations extraordinaires" dans «les chaudes nuits étoilées", de ressentir "la fièvreuse respiration de notre terrible patrie originelle ${ }^{20}$. En ce sens, le héros est moins le compatriote du narrateur que des gens au milieu desquels il vit depuis de nombreuses années, dans un pays étranger, et de ceux qui ont vécu des siècles et des millénaires

20. IV, p. 137: "gorjačečnoe dyhanie nasej strašnoj Prarodiny». 
avant lui. Cette véritable "fraternité», qui transcende le pouvoir du temps, est la réalisation de la formule philosophique de Bounine: "Il n'existe pas dans le monde d'âmes différentes, ni de temps » ${ }^{21}$. Et l'exotisme n'est pas capable de rendre à l'homme la sensation du paradis, c'est-à-dire la perception originelle de soi dans le monde: l'Anglais, héros de "Frères", échoue à recréer, dans ce paradis qu'est Ceylan, «l'amour et la joie de l'existence». L'homme tout entier est dans la sauvegarde ou dans la perte de son sens de l'état originel: «Je vois, j'entends, heureux. Tout est en moi ${ }^{22}$.

Zotov percevait vaguement ce qui se ressent pleinement comme un type particulier de personnes:

[...] que l'on appelle poètes ou artistes. De quoi doivent-ils être doués? De la faculté de sentir avec une force particulière non seulement leur époque, mais aussi une époque étrangère, une époque passée, non seulement leur pays, leur tribu, mais aussi d'autres pays et tribus, des pays et tribus étrangers, non seulement eux-mêmes, mais aussi les autres... ${ }^{23}$

Cette capacité est en fait cette «réminiscence» particulière, «résonnant religieusement dans tout notre être", "vivant dans notre sang, nous liant mystérieusement avec les dizaines et centaines de générations de nos pères qui vécurent en plus d'avoir simplement été», comme l'écrit Bounine dans son article "L'Inonie et Kitège" ${ }^{24}$. Cette "réminiscence» apparaît pour lui comme la qualité principale de la connaissance artistique du monde.

Du point de vue de Bounine, cette "Mémoire imagée (sensible)" se fonde sur la capacité humaine de ressentir son état originel, c'est-àdire de se rappeler intérieurement Adam et de retrouver la sensation de «la douceur du monde divin" comme une perception du monde

2I. I, p. 315: «Net v mire raznyh duš i vremeni v nem net.»

22. I, p. 274: "Ja vižu, slyšu, sčastliv. Vse vo mne.»

23. IV, p. 438: «...kotoryh nazyvajut poètami, hudožnikami. Čem oni dolžny obladat'? Sposobnost'ju osobenno sil'no čuvstvovat' ne tol'ko svoe vremja, no i čužoe, prošloe, ne tol'ko svoju stranu, svoe plemja, no i drugie, čužie, ne tol'ko samogo sebja, no i pročih".

24. "vospominanie», "religiozno zvučaščee vo vsem našem suščestve», "živuščee v krovi, tajno svjazujuščee nas s desjatkami i sotnjami pokolenij naših otcov, živših, a ne tol'ko suščestvovavših", I. Bunin, Okajannye dni. Vospominaja. Stat'i, p. 369. 
véritablement paradisiaque. Iouri Maltsev suppose que le principal stimulus aux voyages de Bounine dans les pays d'Orient était la "quête du paradis perdu ${ }^{25}$; cependant, il limite cette notion à des caractéristiques spatio-temporelles. Bounine trouve effectivement un tel "paradis géographique» et "historique» dans les pays d'Orient, où "tout est sauvage et splendide, comme en Eden ${ }^{26}$.

Pourtant, ce n'est qu'aux jours du «cataclysme russe de 1917 » (Georges Nivat) que Bounine prend conscience du fait que son "paradis» terrestre incontestable est la Russie dans l'unité de ses traits naturels et historiques, spirituels et culturels. C'est la raison de la présence dans son poème "Le Paradis perdu» («Uterjannyj raj», 1919) $\mathrm{d}$ 'intonations amèrement ironiques (et dans une certaine mesure, autoironiques) envers le peuple russe exilé du paradis après qu'il s'est abandonné à la tentation de «la révolution sociale», à l'instar des ancêtres de l'humanité:

Sous les murs défendus du paradis,

A l'heure de midi,

Adam, avec sa femme Eve, est affligé ${ }^{27}$.

Dans ce poème politique apparaît, sans doute, le "paradis» le plus exotique de Bounine. C'est un paradis éclatant de loubok ${ }^{28}$, comme recopié par le poète d'un bouquin populaire bon marché, qui illustre comment "Le Seigneur Dieu planta un paradis en Eden» ${ }^{29}$. De là, les «noirs cyprès", trois fois répétés dans une forme sciemment incorrecte (litt. kuparis), dans le but de renforcer ce côté art populaire; des paons sur les murs du paradis:

Leur queues resplendissent de vert

Leurs petites têtes en des corolles dentelées [...]

Oiseaux fatidiques

Aux yeux merveilleux et menaçants,

25. J. Mal'cev, Ivan Bunin. 1870-1953, p. 40.

26. I, p. 321.

27. I, p. 363: «U rajskoj zapretnoj steny / V čas poludennyj, / Adamij s ženoj Evvoj skorbit."

28. Image d'Epinal russe (N.d.T.).

29. V. Dal', Tolkovyj slovar' živogo velikorusskogo jazyka, p. 56. 
Aux voix angéliques,

Aux beautés féminines... ${ }^{30}$

Pour Bounine se révèlent, comme autant d'attributs essentiels de ce "paradis" exotique, "La blanche église apostolique, / Le blanc sanctuaire aux coupoles dorées, / Le monastère paternel...». C'est précisément dans ces jours fatidiques pour l'histoire russe que Bounine prend conscience de la Russie chrétienne, spirituelle comme d'un "paradis perdu ", alors que la Russie nouvelle, révolutionnaire, apparaît sous les traits de la "pécheresse», engendrant "sa nouvelle progéniture»"des Caïns russes", qu'il décrit avec ire et douleur dans son poème " $\mathrm{A}$ la Russie» («Rossii », 1922), une transposition poétique du psaume 136 «Sur les fleuves de Babylone».

Au même moment, dans les "Jours maudits de la Russie", Bounine conserve sa perception artistique du monde, unique, particulière, dont la base est la "suavité du monde divin", une base qu'il est impossible de perdre même lorsque «la joie de vivre est tuée par la guerre, la révolution " ${ }^{31}$. Ce paysage de la terre russe, absolument privé d'exotisme, est sans doute la seule chose qui n'est pas exposée au cataclysme social, c'est pourquoi le héros de Bounine tente de conserver, en ces jours de tempête historique, sa participation à la vie de la nature, comme moyen de s'élever au-dessus de ce qui est terrestre et transitoire:

O joie des teintes! Encor et encor

L'azur par l'éclatant jardin jaune

Brûle si admirablement, lilas,

Comme des anges qui regarderaient.

O joie des joies! Non - je sais,

Non - je crois que Toi, Seigneur,

Tu ramèneras au paradis perdu

Mes tourments et mes rêves! ${ }^{32}$

3o. I, p. 363-364.

3I. VI, p. 394.

32. I, p. 355: «O radost’ krasok! Snova, snova / Lazur' skvoz' jarkij želtyj sad / Gorit tak divno i lilovo, / Kak budto angely gljadjat. // O radost' radostej! Net, znaju, / Net, verju, gospodi, čto ty / Verneš’ k poterjannomu raju / Moi tomlen’ja i mečty!». 
L'Occident, où Bounine dut vivre de longues années en émigration, ne fut considéré par lui ni comme une patrie, ni comme un ailleurs: la patrie comme l'ailleurs étaient demeurés en Russie. La nature du Sud de la France, que l'écrivain parcourut souvent, ne devint jamais un objet de représentation poétique; c'est à peine si le mistral, portant toujours chez Bounine le froid et la mort, apparaît dans sa poésie et sa prose tardive comme une sorte d'image exotique. Cela dit, son regard cherchait quelque chose de familier dans l'altérité, et, dans un de ses derniers poèmes "Nel mezzo del camin di nostra vita" (1947), revient de nouveau la perception de la «douceur du monde divin», cette perception qu'offre à Bounine la nature désormais italienne et non russe:

Jours d'avril près de Naples,

Quand il fait froid et humide,

Si doux au cœur le monde divin...

Les jardins ont rosi dans les vallées,

Une nuée azurée y demeurait,

Bourgades noires en silence,

Les saules gris saillaient,

Inspirant en somnolant l'opium

De la terre labourée et du fumier;

La menace sombre se cachait

Dans la toison drue et fumante

Dévalant les monts en nuages

Sur leurs escarpements bleutés...

O jours dont je me souviendrai éternellement! ${ }^{33}$

Les éléments exotiques apparaissent ici grâce à l'initiation des images de Dante de la Divine Comédie: "vallée», "monts», "escarpements bleutés", un paysage matinal, absolument reconnaissable est mis en place, comme lorsque "avec joie tout respirait alentour " ${ }^{34}$, anticipant l'introduction du héros de Dante en enfer. En même temps, Bounine cite des lignes de ses propres poèmes programmatiques: «Il fait encore

33. I, p. 375: «Dni bliz Neapolja v aprele, / Kogda tak holoden i syr, / Tak sladok serdcu Božij mir... / Sady v dolinah rozoveli, / V nih goluboj stojal tuman, / Selen'ja černye molčali, / Rakity serye torčali, / Vdyhaja v polusne durman / Zemli razrytoj i navoza. / Tailas' hmuraja ugroza / V dymiaščemsja gustom rune, / Kakim v gorah spuskalis' tuči / Na ih sinejuščie kruči... / Dni večno pamjatnye mne!»

34. A. Dante, Božestvennaja komedija, p. 12. 
froid et humide» (1901) - "Quand il fait froid et humide» (1947), «De nouveau suave le monde divin" (1917) - «Si suave au cœur le monde divin» (1947). Au niveau des motifs, émergent les images du poème «Un Printemps russe» (1905): «En nuée, doucement, dans la steppe» (1905) - «Une Nuée azurée y demeurait» (1947), "Dans un hameau de steppe endormi" (1905) - "Les saules gris saillaient, / Inspirant en somnolant l'opium» (1947), "En fumier de cheval détrempé / Dans la nuée perce le noir chemin» (1905) - «De la terre labourée et du fumier» (1947). A travers le paysage de Dante - italien, exotique - perce le printemps russe, et tout le "tableau" acquiert, dans le texte de Bounine, ces teintes naturelles et vivantes dans lesquelles «luisent l'amour et la joie de l'existence». Ainsi, le "Livre de la nature», écrit par le Créateur Luimême ${ }^{35}$, "est relu» par les créateurs de culture, capables de ressentir et de reproduire artistiquement la force sainte de l'amour de Dieu.

Le titre de ce poème et son contenu semblent entrer en contradiction: l'idée courante selon laquelle la vie déclinante voit se perdre la «joie de l'existence» ne correspond pas à l'affirmation lyrique du héros sur la «douceur au cœur du monde divin». Il est possible que cette contradiction soit ironique; le poème orienté vers la formule dantéenne sur la vie terrestre et sur la perte du "droit chemin", l'«égarement» dans les passions. Discrètement polémique contre Dante (dont la formule conduit inexorablement le pécheur vers l'enfer), Bounine écrit à nouveau sur la "douceur" paradisiaque de l'existence; il rend ainsi le "paradis perdu " à sa jeunesse, à sa Russie, à sa poésie.

De cette manière, tous les aspects de l'exotisme dans l'œuvre de Bounine - sacré, géographique, colonial, culturel - n'en font pas une caractéristique distinctive de sa vision du monde et de sa poétique. La puissance artistique de Bounine, ses convictions spirituelles et religieuses, ainsi que sa position politique bien définie, le rendent capable de percevoir l'étranger comme le sien propre (et, pendant la révolution, le sien propre comme l'étranger), le lointain (dans le temps et l'espace) comme proche, l'exotique comme fraternellement familier.

Olga Berdnikova
Voronèje

35. K. (Prot.) Kopejkin, «Kniga prirody v vostočno- i zapadnohristianskoj tradicii», p. 208. 


\section{BIBLIOGRAPHIE}

Ajhenval'D, J., Siluèty russkih pisatelej, Moskva, 1994.

Bunin, I., Sobranie sočinenij v 6 tt., t. 3, Moskva, 1987.

—, Okajannye dni. Vospominaja. Stat'i, Moskva, 1990.

—, "Mašinopis' s avtorskoj pravkoj", Orlovskij gosudarstvennyj muzej

I. S. Turgeneva. Arhiv Bunina. Inv. 2736.

Čunovsкij, K. I., "Smert', krasota i ljubov' v tvorčestve I. Bunina», Niva, 49 (1914).

DAL', V., Tolkovyj slovar' živogo velikorusskogo jazyka, Sankt-Peterburg/ Moskva, 1882.

Dante, A., Božestvennaja komedija, Moskva, 2007.

GoR’kiJ, M., Sobr. soč. v 30 tt., Moskva/Leningrad, 1950-1956.

Kopejkin, K. (Prot.), «Kniga prirody v vostočno- i zapadnohristianskoj tradicii ", in Dva grada: dialog nauki i religii: Vostočno- $i$ zapadnoevropejskaja tradicii, Moskva, 2002.

Mal'cev, J., Ivan Bunin. 1870-1953, Frankfurt-am-Main, 1994.

Marullo, T. G., "If You see Buddha», Studies of Ivan Bunin, Illinois, 1998.

Muromceva-Bunina, V. N., Žizn’ Bunina. Besedy spamjat'ju, Moskva, 1989.

SlivickajA, O., "Bunin i Vostok: K postanovke voprosa», in Izvestija Voronežskogo pedagogičeskogo instituta, t. 114, Voronež, 1971.

—, «Povyšennoe čuvstvo žizni», in Mir Ivana Bunina, Moskva, 2004.

Smol’Janinova, E., "Vostočnye motivy v rannej proze I. A. Bunina», in I. A. Bunin v dialoge èpoh, Voronež, 2002.

TJan' Hunmin', "Sopostavlenie rasskazov “Sosny" i “Brat'ja” s točki zrenija buddizma», I. A. Bunin v načale XXI veka, Voronež, 2005. 
